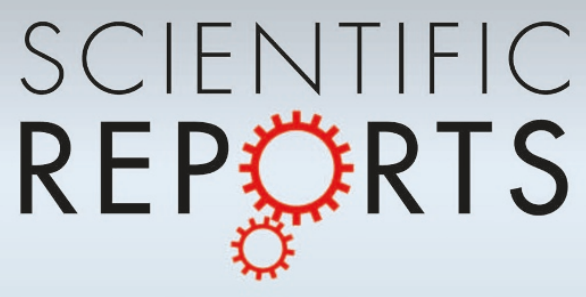

OPEN

SUBJECT AREAS:

STATISTICAL PHYSICS

NONLINEAR PHENOMENA

COMPLEXITY

POPULATION DYNAMICS

Received

17 June 2013

Accepted

6 December 2013

Published

8 January 2014

Correspondence and requests for materials should be addressed to L.W. (fdlwang@gmail. com) or Z.W. (zhenwang0@gmail. com)

\section{Freezing period strongly impacts the emergence of a global consensus in the voter model}

\author{
Zhen Wang' , Yi Liu' ${ }^{2}$ L Lin Wang ${ }^{3,4}$, Yan Zhang ${ }^{3}$ \& Zhen Wang ${ }^{5,6,7}$
}

'School of Software, and Computational Social Science Laboratory, School of Innovation Experiment, Dalian University of Technology, Dalian 1 16621, China, ${ }^{2}$ Department of Public Management, School of Public Administration and Law, Dalian University of Technology, Dalian 116024 , China, ${ }^{3}$ Adaptive Networks and Control Laboratory, Department of Electronic Engineering, Fudan University, Shanghai 200433, China, ${ }^{4}$ Centre for Chaos and Complex Networks, Department of Electronic Engineering, City University of Hong Kong, Hong Kong SAR, China, ${ }^{5}$ Department of Physics, Hong Kong Baptist University, Hong Kong SAR, China, ${ }^{6}$ Center for Nonlinear Studies, and the Beijing-Hong Kong-Singapore Joint Center for Nonlinear and Complex Systems (Hong Kong), Hong Kong Baptist University, Hong Kong SAR, China, ${ }^{7}$ Institute of Computational and Theoretical Studies, Hong Kong Baptist University, Kowloon Tong, Hong Kong SAR, China.

It is well known that human beings do not always change opinions or attitudes, since the duration of interaction with others usually has a significant impact on one's decision-making. Based on this observation, we introduce a freezing period into the voter model, in which the frozen individuals have a weakened opinion switching ability. We unfold the presence of an optimal freezing period, which leads to the fastest consensus, using computation simulations as well as theoretical analysis. We demonstrate that the essence of an accelerated consensus is attributed to the biased random walk of the interface between adjacent opinion clusters. The emergence of an optimal freezing period is robust against the size of the system and the number of distinct opinions. This study is instructive for understanding human collective behavior in other relevant fields.

U nderstanding the reason how a macroscopically ordered state (e.g., consensus) can emerge among selforganized interactive individuals is one of the open questions in natural and social sciences, which attracts much attention by the scientific communities from diverse disciplines. Various experimental and theoretical frameworks have been proposed from the viewpoints of statistical physics, economics and sociology $y^{1-15}$. In particular, one simple yet paradigmatic dynamical process is opinion formation dynamics.

Through the development of several decades, dozens of spin-like models have been put forward to study the evolution of opinion dynamics. Typical examples include the voter model ${ }^{16,17}$, Sznajd model ${ }^{18}$, majority rule model $^{19-21}$, and bounded confidence model ${ }^{9,22,23}$, to mention a few. Among them, the voter model has attracted the most notable attention ${ }^{24-30}$. In its basic version, each individual (voter) possesses one of two opinion states, denoted by +1 or -1 . At each time interval, a randomly selected voter adopts the opinion of one of his neighbors, who is also chosen randomly. In this sense, the transition rate of one's opinion is proportional to the fraction of the opposite attitude in his neighborhood. The dynamical process is iterated until it reaches a stable state. Although being composed of such a simple mechanism, the voter model produces rich dynamical behaviors ${ }^{31-38}$.

A series of extended models were proposed to promote the study of collective opinion behavior, such as the vacillating voter model ${ }^{39}$, nonlinear voter mode ${ }^{40}$, heterogeneous voter mode ${ }^{41,42}$, and constrained voter model ${ }^{43}$. More specifically, introducing the 'zealot-voters' who never changed opinion, Refs. 8,44 found that the phenomenon of consensus was not an asymptotic outcome of repeated elections in democratic societies. Refs. 45, 46 unveiled the significant effect of social influence and heterogeneous beliefs on the evolution of public opinions. It is worth emphasizing that present voter models generally assume that the individuals' behavior is a Markovian memoryless pattern, which means that per unit time, the decision-making of voters depends only on the status of their neighbors at the current time step. While in the realistic life, the non-Markovian process is ubiquitous as well ${ }^{47}$. For example, in recent studies ${ }^{48,49}$, it was found that non-Markovian patterns of epidemic spreading dramatically impact the threshold of disease outbreak. Along a similar way, if the non-Markovian factor is introduced into the voter model, how does the evolution outcome fare? To sketch a comprehensive picture on this issue, Refs. 25, 26 studied the effect of memory-dependent transition rates, where the opinion changing was designated a certain inertia, determined by the persistence time of one's current opinion. The longer a voter 
maintained his opinion, the less probable that he would change it in the long run. They found that the process towards reaching a macroscopic consensus was accelerated by slowing down the microscopic dynamics.

In realistic systems, there also exists the opposite scenario, where the opinion switching rate of individuals increases with the persistence time. In this case, the longer an agent sustains his current state, the higher transition possibility he has. For example, Ref. 50 introduced a time-dependent learning capability into the dynamics of evolutionary game, which showed that this simple yet meaningful mechanism was able to maintain the diversity of strategies. Moreover, similar assumptions of a predefined latent or infectious period has often been adopted in the epidemiological models ${ }^{51-56}$. Inspired by these aspects, an interesting question poses itself, which we aim to address in what follows. Namely, if the transition rate increases with the persistence time, will it be beneficial to the consensus formation?

Here, we introduce the freezing period into the voter model, in which frozen individuals have a small transition rate for switching opinions. Through extensive computational simulations, we study the impact on the emergence of a global consensus. We unveil that the freezing period has an optimal value, which leads to the fastest consensus. To verify this finding, theoretical analysis is provided as well.

\section{Results}

The effect of freezing period: Basic simulation results. The freezing period $H$ can be regarded as a latent period, which determines the switching capacity of a voter's opinion (see Methods for further details). To begin with, we study its effect on the consensus time $T_{c}$. Figure 1 illustrates the dependence of consensus time $T_{c}$ on $H$ for different lattice sizes of $N$. It is evident that the variance of $T_{c}$ displays a non-monotonous pattern as the freezing period $H$ changes, regardless of the specific value of $N$. With a small $H$, the impact on $T_{c}$ is marginal, since the system still requires significantly more time to reach the final consensus. As the freezing period gradually increases, there emerges an optimal value of $H$, which produces the fastest consensus formation. This characteristic value is denoted as $H_{o p t}$. When we further increase the freezing period $H$, the efficiency of reaching a consensus is exacerbated. The presence of $H_{o p t}$ significantly reduces the time for reaching the final global consensus. The impact of lattice size $N$ on $H_{o p t}$ is also investigated. In the inset of Figure 1, one can find that $H_{o p t}$ gradually increases with the growth of $N$, which implies that $H_{o p t}$ should be enlarged to guarantee the shortest consensus time as the lattice size increases. This is similar to what has been mentioned in Refs. 22, 33, 57-59.

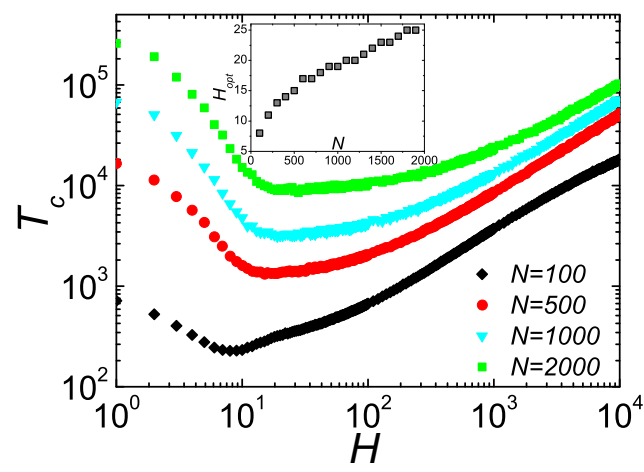

Figure $1 \mid$ Consensus time $T_{c}$ vs. $H$ for different values of lattice size $N$. Note that there presents an optimal value of $H$, named $H_{o p t}$, producing the shortest consensus time, regardless of the values of $N$. The inset shows the variance of $H_{\text {opt }}$ as dependent on the lattice size $N$. We fix $q=0.01$.
To verify the effect of $H_{\text {opt }}$ on accelerating the emergence of a global consensus, we examine the time courses of opinion formation with several typical values for $H$. It is pertinent that some parameters are defined first to simplify the discussion. $P_{\sigma}(t)$ is denoted as the overall number of voters possessing the opinion $\sigma$ at time $t$, and thus the average opinion (or 'magnetization'38) is $M(t)=\frac{1}{N} \mid P_{+}(t)-$ $P_{-}(t) \mid$. Figure 2 shows the evolution of $M(t)$ with several typical values for $H . H=0$ returns to the traditional scenario, where the transition of opinion is fluctuation-driven. In this case, the slow increment of $M(t)$ leads to a sufficiently long transient time to reach the final ordered state. Given a very large freezing period $H$ (e.g., $H=$ $4,000)$, the initial state of the system will be locked into a frozen phase, in which voters hardly change their original opinions. When the persistence time exceeds this freezing period, $M(t)$ rapidly increases, which implies that the system quickly proceeds towards the final consensus. The consensus time $T_{c}$ in the scenario of $H=4$, 000 is almost equivalent to that of the standard scenario $\left(T_{c}>3 *\right.$ $\left.10^{4}\right)$. Strikingly, when $H=15$, the evolution towards a consensus will be dramatically accelerated. In the early stage, the probability of opinion switching is still very small, due to the introduction of a freezing period. However, when $t>H_{o p t}$, the increment of $M(t)$ suddenly accelerates. The system only uses about 1,700 time steps to reach the final consensus, which is much faster than what one can expect for the other cases. The presence of $H_{\text {opt }}$ warrants the most efficient condition for the consensus emergence.

The reason for the accelerated consensus: Biased random walk. Figure 3 shows the snapshots of the evolution process with three typical values for $H$. As shown in Figure 4, under the scenario of $H$ $=0$, the number of opinion clusters, $N_{o c}$, decreases as time proceeds. In particular, in the first $10^{2}$ time steps, the reduction of $N_{o c}$ is the fastest. In this standard voter model, the interface between adjacent opinion clusters performs a random walk, and vanishes once it encounters another one. The disadvantage of this mechanism is especially evident when only two giant opinion clusters remain in the system, since their unremitting competition remarkably postpones the formation of a global consensus. As shown in Figure 3(a) and Figure 4, the two final giant clusters emerge at about $10^{4}$ th time step, whereas the system spends almost $3 * 10^{4}$ time steps for reaching the final consensus.

When the freezing period increases to $H_{o p t}$, the dynamic feature changes dramatically. As shown in Figure 3(b) and Figure 4, after voters escape from the initial freezing period, the number of opinion clusters $N_{o c}$ is quickly decreased. In this case, the interface separating adjacent opinion clusters does not merely perform a random walk, but rather takes a biased random walk. For further explanation, we use an example to illustrate the movement of a typical interface in

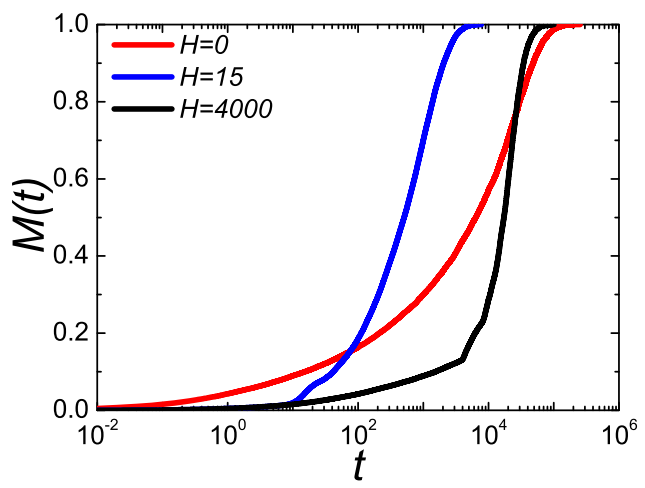

Figure $2 \mid$ Time courses characterizing the evolution of magnetization $M(t)$ with different values of $H$. Although the initial value of $M(t)$ is small under the scenario of $H=15$, it requires the shortest time to reach a global consensus. We fix $N=500, q=0.01$. 


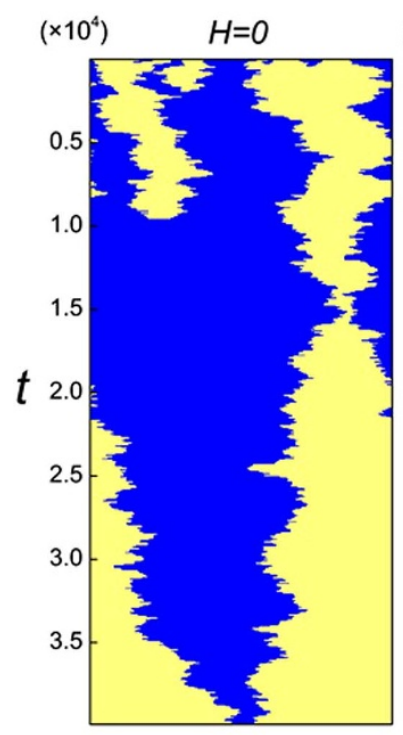

(a)

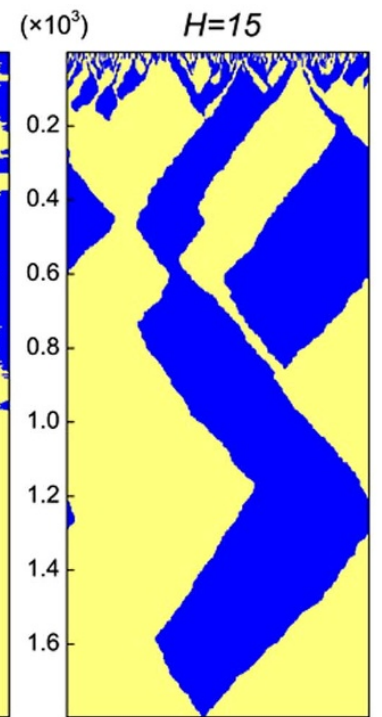

(b)

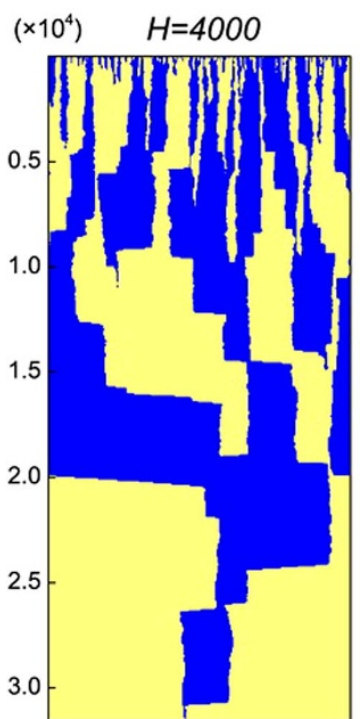

(c)

Figure 3 Snapshots for the evolution of opinion dynamics with three typical freezing periods. From left to right, the values of $H$ are 0,15 and 4000 . In each panel, the vertical axis corresponds to the elapsed time, while the yellow and blue areas on the horizontal lines represent the opinion clusters. It is evident that the total time spent to reach a global consensus under the scenario of $H=15$ is far less than what we can expect for the other two cases. We fix $N=500, q=0.01$.

Figure 5. For simplicity, the capital letter $A(B)$ represents the voters having opinion $+1(-1)$, while the lowercase letters $a, b, c, d, e, f$ denote the positions of voters on the lattice. Given that the persistence time of each voter on the left of the interface (located at the positions $a, b, c$ in Figure 5(a)) is larger than the freezing period (i.e., $\tau_{a(b, c)}>H$ ), and the right-hand voter closest to the interface (located at the position $d$ in Figure 5(a)) has a persistence time smaller than the freezing period (i.e, $\tau_{d}<H$ ), the interface has a high probability to gradually move leftward (see Figure 5(a) $\rightarrow$ Figure 5(b)). Occasionally, some special situations may occur: (i) the left-hand voter closest to the interface (located at the position $c$ in Figure 5(a)) does not change his opinion, yet the voter at the position $d$ accepts the left-hand neighbor's opinion (see Figure 5(a) $\rightarrow$ Figure 5(c)); (ii) two voters closest to the interface (located at the positions $c, d$ in Figure 5(a)) do not change their opinions until their persistence time both reaches $H$, while the right-hand voter closest to the interface finally adopts the left-hand neighbor's opinion. In these cases, the movement of the interface will shift its direction with a very small probability, since: (i) the parameter $q$ is assigned a tiny value, (ii) the probability is quite small for that a pair of neighbors with distinct opinions maintain their respective choices for about $H$ steps.

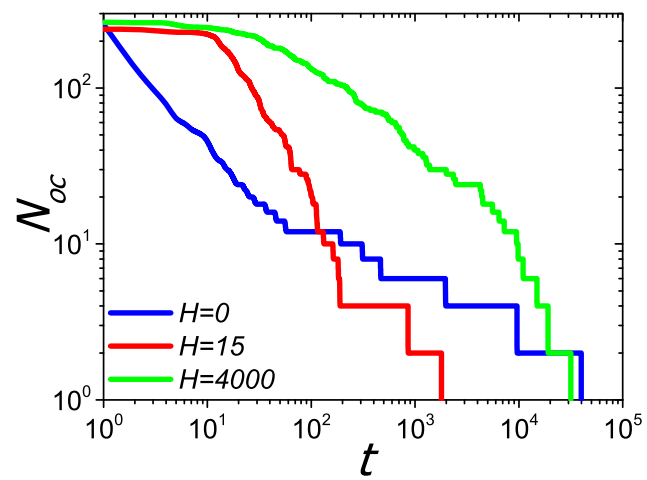

Figure $4 \mid$ Time courses of opinion clusters $N_{o c}$ for different values of $H$. It is obvious that $N_{o c}$ quickly decreases to 1 when $H=15$ (after voters pass the initial freezing period), which is much faster than the results of other cases. We fix $N=500, q=0.01$.
With an optimal freezing period, $H_{o p t}=15$, the effect of a biased random walk not only accelerates the merging of clusters at the early stage, but also dramatically reduces the time wasted by the competition between the two final clusters (see Figure 3(b)). However, a further increment of $H$ weakens this advantage. As an illustration, we show the evolution snapshot of the scenario $H=4,000$ in Figure 3(c). In this case, most interfaces are located between the opinion clusters with persistence times smaller than $H$. For any interface, only when either side of the cluster escapes from the initial freezing period, a biased random walk occurs. Therefore, the global consensus is largely deferred. To further verify the presence of optimal freezing period $H_{o p t}$, a simple theoretical analysis based on the diffusive process is supplied in Supporting Information.

The influence of the overall number of opinions $G$ and the switching ability $\boldsymbol{q}$. At last, it remains of interest to examine the impact of the overall number of opinions $G$ and the switching ability $q$ on the consensus time $T_{c}$. Figure 6 shows the dependence of $T_{c}$ on $G$. It is obvious that $T_{c}$ gradually increases with the increment of $G$. Moreover, as shown in the inset of Figure 6, the optimal freezing

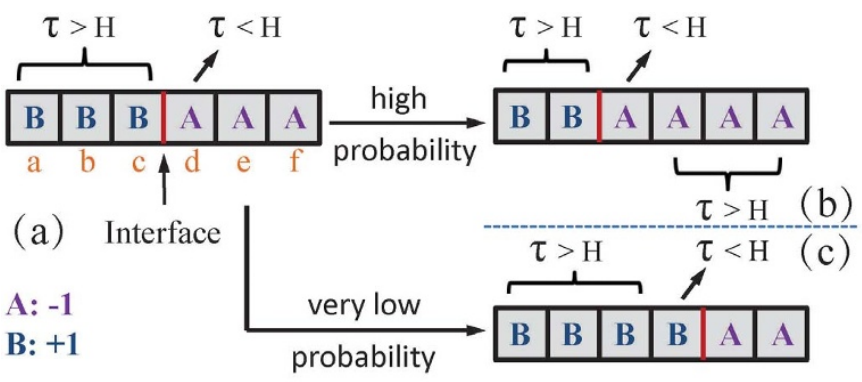

$\tau>\mathrm{H}$

Figure $5 \mid$ A schematic illustration of the biased random walk for a typical interface between neighboring opinion clusters. For simplicity, the capital letters $A, B$ represent the voters with opinion +1 or -1 , respectively, while the lowercase letters $a, b, c, d, e, f$ denote the positions of voters on the lattice. In each panel, the red line highlights the interface, which separates the clusters of different opinions. 


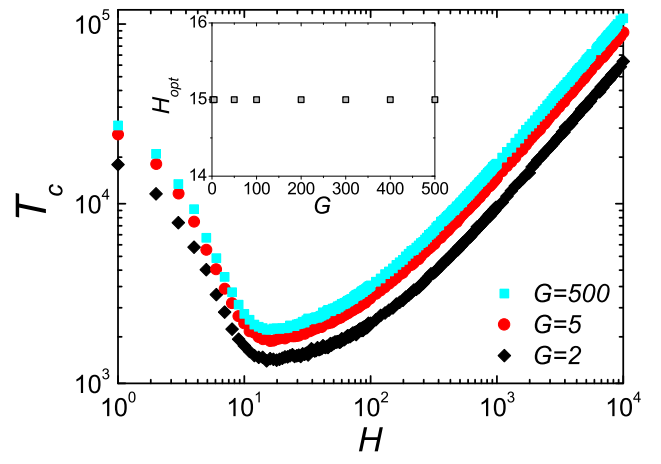

Figure 6 Consensus time $T_{c}$ vs. $H$ for different number of opinions $G$. Note that there always exists an optimal freezing period, $H_{o p t}$, producing the shortest time to reach the final consensus, regardless of the values of $G$. The inset shows the variance of $H_{o p t}$ as dependent on $G$. We fix $N=500, q$ $=0.01$.

period $H_{\text {opt }}$ remains unchanged with the increment of $G$. Therefore, the value of $H_{o p t}$ is robust, regardless of the changes in $G$. Figure 7 presents the relevance between $T_{c}$ and $H$ for different values of $q$. In the limit $q \rightarrow 0$, the scenario of $H_{o p t}$ produces the shortest consensus time. However, increasing the value of $q$, this non-monotonous phenomenon becomes more inconspicuous.

To give a holistic profile, the $(q, H)$ phase diagram of the consensus time $T_{c}$ is shown in Figure 8 . With respect to the Markovian scenarios (i.e., $q=1$ or $H=0$ ), there always exists an optimal freezing period $H_{o p t}$, which leads to the fastest consensus, when $q$ is small. The smaller the value of $q$, the faster the formation of a global consensus with $H_{o p t}$.

\section{Discussion}

In summary, we have introduced the freezing period into the voter model. Through extensive computational simulations, we unfold that there presents an optimal freezing period, denoted by $H_{o p}$, guaranteeing the fastest consensus, which is robust against the changing of lattice size and the overall number of opinions. In order to explain the boosting effect of $H_{o p t}$, we have examined the time courses of opinion clusters, and unveiled that this achievement is attributed to the mechanism of biased random walks of the interfaces that separate neighboring opinion clusters. Moreover, we have theoretically analyzed the diffusion rate of interfaces, which verifies that the presence of $H_{o p t}$ does guarantee the most effective transmission of opinions.

Examples of the freezing period are prevalent in various natural contexts, such as the creation of new technologies in the field of telecommunications. In this sense, we wish that this work will inspire

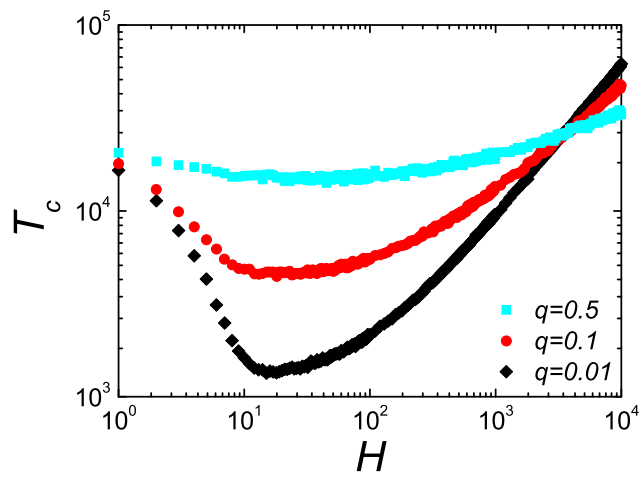

Figure 7 Consensus time $T_{c}$ vs. $H$ for different values of switching ability $q$. With the increment of $q$, the shortest consensus time $T_{c}$ increases as well. We fix $N=500$.

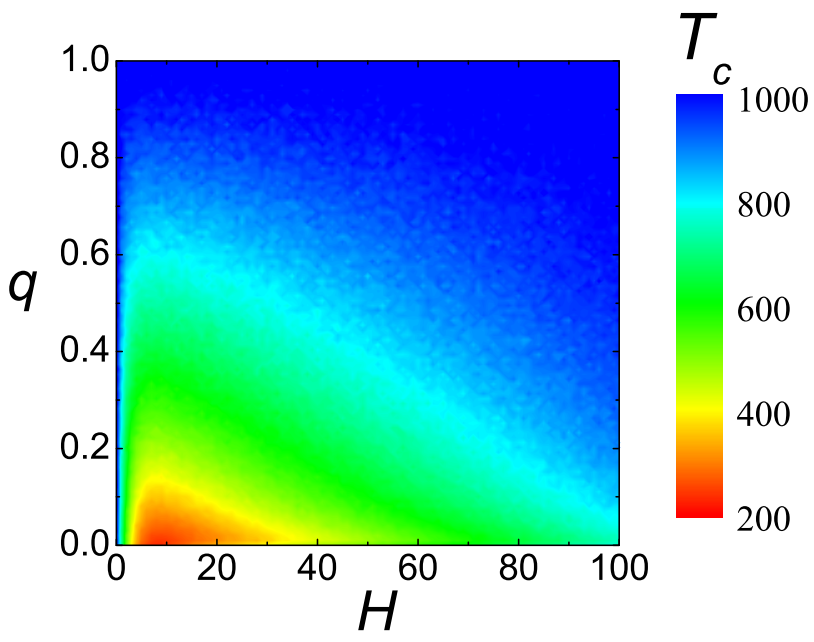

Figure $8 \mid(q, H)$ phase diagram of the consensus time $T_{c}$. We fix $N=500$.

further studies to improve the understanding on opinion formation dynamics in other relevant models, and to extend the study to complex networked topologies.

\section{Methods}

Model definition and algorithm details. We consider the voter model with $N$ voters allocated on the nodes of a regular one-dimensional lattice, with the periodic boundary condition. Initially, each voter $i$ is designated one of two opinions, $\sigma_{i}=+1$ or -1 , with equal probability. Since we will also study cases with more than two opinions, the parameter $G$ is introduced to describe the overall number of distinct opinions. Voters update their opinions as follows: a voter $i$ is selected at random, and will be affected by one of his neighbors, $j$, who is also chosen randomly. If they have the same opinion, $i$ preserves his viewpoint; if their opinions are distinct, $i$ adopts the opinion of $j$ with the probability $\omega_{i}^{V}\left(\sigma_{i} \mid \sigma_{j}, t\right)$. When such a updating event takes place for $N$ times, the time step is increased by one. In the standard voter model, the value of $\omega_{i}^{V}\left(\sigma_{i} \mid \sigma_{j}, t\right)$ equals to $1^{26,28,29}$. In our model, the transition of opinions is not only affected by neighbors' opinions, but is also determined by the persistence time of opinions.

To account for the effect of opinion persistence time, we introduce the freezing period $H$, in which voters are frozen with lowered opinion switching ability. The modeling procedure is iterated with the elementary steps: (i) randomly pick a voter $i$; (ii) the voter $i$ accepts the opinion of a randomly chosen neighbor $j$ with the probability

$$
\omega_{i}^{V}\left(\sigma_{j} \mid \sigma_{i}, t\right)= \begin{cases}q & \text { if } \tau_{i}<H \\ 1 & \text { if } \tau_{i} \geq H\end{cases}
$$

where the parameter $q(0 \leq q \ll 1)$ represents the weakened switching ability during the freezing period, and the persistence time $\tau_{i}$ registers the elapsed time since the last change of $i$ 's opinion. When $H=0$, the traditional model is recovered, where all players have uniform, time-independent switch capacity; while for $H>0$, the nonMarkovian effect is introduced. As in Refs. 25, 26, 50, we assume that (i) at the beginning $(t=0)$, and at each time step immediately after the opinion change of voter $i, \tau_{i}$ is reset to zero (i.e., $\tau_{i}=0$ ); (ii) if voter $i$ does not change his opinion, his persistence time is increased by one (i.e., $\left.\tau_{i}=\tau_{i}+1\right)$. Therefore, once the persistence time $\tau_{i}$ is smaller than the freezing period $H$, the switching rate of $i$ is decreased to $q$; when $\tau_{i} \geq H$, voter $i$ recovers to the standard case. Moreover, we have also certified that the initial distribution of the elapsed time (e.g., exponential or power-law distributions) does not change the main results.

The elementary steps are iterated until the system reaches a state of consensus. In a full time step, on average, each voter has a chance to update his opinion once. The consensus time $T_{c}$ is defined to capture the time spent in reaching the global consensus. In addition, since Refs. 57, 58 showed that $T_{c}$ is related to the size of the system, the simulation results are averaged up to $10^{4}$ independent realizations for each set of parameters.

1. Boccaletti, S., Latora, V., Moreno, Y., Chavez, M. \& Hwang, D. U. Complex networks: Structure and dynamics. Phys. Rep. 424, 175-308 (2006).

2. Arenas, A., Díaz-Guilera, A., Kurths, J., Moreno, Y. \& Zhou, C. Synchronization in complex networks. Phys. Rep. 469, 93-153 (2008).

3. Barrat, A., Barthélemy, M. \& Vespignani, A. Dynamical Processes on Complex Networks. (Cambridge University Press, Cambridge, U.K., 2008).

4. Dorogovtsev, S. N., Goltsev, A. V. \& Mendes, J. F. F. Critical phenomena in complex networks. Rev. Mod. Phys. 80, 1275-1335 (2008). 
5. Perc, M. \& Szolnoki, A. Coevolutionary games-A mini review. Biosystems 99, 109-125 (2010).

6. Redner, S. A Guide to First-Passage Processes. (Cambridge University Press, Cambridge, U.K., 2001).

7. Castellano, C., Fortunato, S. \& Loreto, V. Statistical physics of social dynamics. Rev. Mod. Phys. 81, 591-646 (2009).

8. Mobilia, M. Does a Single Zealot Affect an Infinite Group of Voters? Phys. Rev. Lett. 91, 028701 (2003).

9. Hegselmann, R. \& Krause, U. Opinion dynamics and bounded confidence: models, analysis and simulation. Journal of Artifical Societies and Social Simulation (JASSS) 5, 3 (2002)

10. Fowler, J. H. \& Dawes, C. T. Two Genes Predict Voter Turnout. Journal of Politics 70, 579-594 (2008)

11. Szabó, G. \& Fáth, G. Evolutionary games on graphs. Phys. Rep. 446, 97-216 (2007).

12. Durrett, R. et al. Graph fission in an evolving voter model. Proc. Natl. Acad. Sci. USA 109, 3682-3687 (2012).

13. Salathé, M. \& Bonhoeffer, S. The effect of opinion clustering on disease outbreaks. J. R. Soc. Interface 5, 1505-1508 (2008).

14. Sánchez, A. D., López, J. M. \& Rodríguez, M. A. Nonequilibrium phase transitions in directed small-world networks. Phys. Rev. Lett. 88, 048701 (2002).

15. Galam, S. Local dynamics vs. social mechanisms: A unifying frame. EPL 70, 705 (2005).

16. Holley, R. A. \& Liggett, T. M. Ergodic theorems for weakly interacting infinite systems and the voter model. Ann. Probab. 3, 643 (1975).

17. Suchecki, K., Eguíluz, V. M. \& San Miguel, M. Voter model dynamics in complex networks: Role of dimensionality, disorder, and degree distribution. Phys. Rev. E 72, 036132 (2005).

18. Sznajd-Weron, K. \& Sznajd, J. Opinion evolution in closed community. Int. J. Mod. Phys. C 11, 1157 (2000)

19. Lambiotte, R. How does degree heterogeneity affect an order-disorder transition? EPL 78, 68002 (2007).

20. Krapivsky, P. L. \& Redner, S. Dynamics of Majority Rule in Two-State Interacting Spin Systems. Phys. Rev. Lett. 90, 238701 (2003).

21. Wu, Z. X. \& Holme, P. Majority-vote model on hyperbolic lattices. Phys. Rev. E 81 , 011133 (2010)

22. Kozma, B. \& Barrat, A. Consensus formation on adaptive networks. Phys. Rev. E 77, 016102 (2008)

23. Hu, H. B. \& Wang, X. F. Discrete opinion dynamics on networks based on social influence. J. Phys. A 42, 225005 (2009).

24. Castellano, C., Vilone, D. \& Vespignani, A. Incomplete ordering of the voter model on small-world networks. EPL 63, 153 (2003).

25. Stark, H. U., Tessone, C. J. \& Schweitzer, F. Decelerating Microdynamics Can Accelerate Macrodynamics in the Voter Model. Phys. Rev. Lett. 101, 018701 (2008).

26. Stark, H. U., Tessone, C. J. \& Schweitzer, F. Slower is faster: Fostering consensus formation by heterogeneous inertia. Adv. Complex. Syst. 11, 551 (2008).

27. Sood, V. \& Redner, S. Voter Model on Heterogeneous Graphs. Phys. Rev. Lett. 94 178701 (2005)

28. Dornic, I., Chaté, H., Chave, J. \& Hinrichsen, H. Critical Coarsening without Surface Tension: The Universality Class of the Voter Model. Phys. Rev. Lett. 87, 045701 (2001)

29. Liggett, T. M. Interacting Particle Systems. (Springer-Verlag, New York, 1985).

30. Lambiotte, R., Saramäki, J. \& Blondel, V. D. Dynamics of latent voters. Phys. Rev. E 79, 046107 (2009).

31. Pereira, L. F. C. \& Brady Moreira, F. G. Majority-vote model on random graphs. Phys. Rev. E 71, 016123 (2005)

32. Benczik, I. J., Benczik, S. Z., Schmittmann, B. \& Zia, R. K. Opinion dynamics on an adaptive random network. Phys. Rev. E 79, 046104 (2009).

33. Yang, H. X., Wu, Z. X., Zhou, C. S., Zhou, T. \& Wang, B. H. Effects of social diversity on the emergence of global consensus in opinion dynamics. Phys. Rev. E 80, 046108 (2009).

34. Shao, J., Havlin, S. \& Stanley, H. E. Dynamic Opinion Model and Invasion Percolation. Phys. Rev. Lett. 103, 018701 (2009)

35. Frachebourg, L. \& Krapivsky, P. Exact results for kinetics of catalytic reactions. Phys. Rev. E 53, R3009 (1996).

36. Slanina, F. \& Lavicka, H. Analytical results for the Sznajd model of opinion formation. Eur. Phys. J. B 35, 279 (2003).

37. Suchecki, K., Eguíluz, V. M. \& San Miguel, M. Conservation laws for the voter model in complex networks. EPL 69, 228 (2005).

38. Sabatelli, L. \& Richmond, P. Non-monotonic spontaneous magnetization in a Sznajd-like consensus model. Physica A 334, 274 (2004).

39. Lambiotte, R. \& Redner, S. Dynamics of vacillating voters. J. Stat. Mech 10, L10001 (2007).

40. Molofsky, J., Durrett, R., Dushoff, J., Griffeath, D. \& Levin, S. Local Frequency Dependence and Global Coexistence. Theor. Pop. Biol. 55, 270 (1999).
41. Masuda, N., Gibert, N. \& Redner, S. Heterogeneous voter models. Phys. Rev. E 82, 010103(R) (2010)

42. Masuda, N. \& Redner, S. Can partisan voting lead to truth? J. Stat. Mech L02002 (2011).

43. Vazquez, F., Krapivsky, P. L. \& Redner, S. Constrained Opinion Dynamics: Freezing and Slow Evolution. J. Phys. A 36, L61 (2003).

44. Mobilia, M. \& Georgiev, I. T. Voting and catalytic processes with inhomogeneities. Phys. Rev. E 71, 046102 (2005).

45. Latané, B. The psychology of social impact. Am. Psychologist 36, 343 (1981).

46. Galam, S. Heterogeneous beliefs, segregation, and extremism in the making of public opinions. Phys. Rev. E 71, 046123 (2005).

47. Balcan, D. \& Vespignani, A. Phase transitions in contagion processes mediated by recurrent mobility patterns. Nat. Phys. 7, 581 (2011).

48. Van Mieghem, P. \& van de Bovenkamp, R. Non-Markovian infection spread dramatically alters the susceptible-infected-susceptible epidemic threshold in networks. Phys. Rev. Lett. 110, 108701 (2013).

49. Wang, L., Wang, Z., Zhang, Y. \& Li, X. How human location-specific contact patterns impact spatial transmission between population? Sci. Rep. 3, 1468 (2013).

50. Szolnoki, A., Perc, M., Szabó, G. \& Stark, H. U. Impact of aging on the evolution of cooperation in the spatial prisoner's dilemma game. Phys. Rev. E 80, 021901 (2009).

51. Pastor-Satorras, R. \& Vespignani, A. Epidemic Spreading in Scale-Free Networks Phys. Rev. Lett. 86, 3200 (2001).

52. Newman, M. E. J. Threshold Effects for Two Pathogens Spreading on a Network. Phys. Rev. Lett. 95, 108701 (2005).

53. Parshani, R., Carmi, S. \& Havlin, S. Epidemic Threshold for the SusceptibleInfectious-Susceptible Model on Random Networks. Phys. Rev. Lett. 104, 258701 (2010).

54. Wang, L., Li, X., Zhang, Y. Q., Zhang, Y. \& Zhang, K. Evolution of Scaling Emergence in Large-Scale Spatial Epidemic Spreading. PLoS ONE 6, e21197 (2011).

55. Wang, L., Zhang, Y., Huang, T. Y. \& Li, X. Estimating the value of containment strategies in delaying the arrival time of an influenza pandemic: A case study of travel restriction and patient isolation. Phys. Rev. E 86, 032901 (2012).

56. Wang, L., Zhang, Y., Wang, Z. \& Li, X. The impact of location-specific contact pattern on the sir epidemic transmission between populations. Int. J. Bifurcat. Chaos 23, 1350095 (2013).

57. Sood, V., Antal, T. \& Redner, S. Voter models on heterogeneous networks. Phys. Rev. E 77, 041121 (2008).

58. Baxter, G. J., Blythe, R. A. \& McKane, A. J. Fixation and Consensus Times on a Network: A Unified Approach. Phys. Rev. Lett. 101, 258701 (2008)

59. Keizo, S. et al. Referring to the social performance promotes cooperation in spatial prisoner's dilemma games. Phys. Rev. E 86, 031141 (2012).

\section{Acknowledgments}

We acknowledge the helpful suggestions from Naoki Masuda. We also thank Nakema Deonauth for his support in preparing this manuscript. This work was partly supported by the National Natural Science Foundation of China (No.71203017, No.61272173, No.61374169), the Key Project of National Social Science Foundation of China (No.12AGL010), and the Fundamental Research Funds for Central Universities (No.DUT12JR08, No.DUT13LK38). L.W. also acknowledges the partial support by Fudan University Excellent Doctoral Research Program (985 Project).

\section{Author contributions}

Z.W., L.W. and Z.W. planned the study; Z.W., Y.L., L.W., Y.Z. and Z.W. performed the experiments; Z.W., L.W. and Z.W. analyzed the data, developed the theory, and wrote the paper.

\section{Additional information}

Supplementary information accompanies this paper at http://www.nature.com/ scientificreports

Competing financial interests: The authors declare no competing financial interests.

How to cite this article: Wang, Z., Liu, Y., Wang, L., Zhang, Y. \& Wang, Z. Freezing period strongly impacts the emergence of a global consensus in the voter model. Sci. Rep. 4, 3597; DOI:10.1038/srep03597 (2014)

This work is licensed under a Creative Commons AttributionNonCommercial-NoDerivs 3.0 Unported license. To view a copy of this license, visit http://creativecommons.org/licenses/by-nc-nd/3.0 